\title{
Origins Space Telescope: trades and decisions leading to the baseline mission concept
}

\author{
David Leisawitz $\odot,{ }^{\mathrm{a}, *, \dagger}$ Edward Amatucci $\odot{ }^{\mathrm{a}}{ }^{\mathrm{a}}$ Lynn Allen, ${ }^{\mathrm{b}}$ \\ Jonathan Arenberg $\odot,{ }^{c}$ Lee Armus, ${ }^{d, \dagger}$ Cara Battersby $\odot,{ }^{\text {e }, \dagger}$ James Bauer,,${ }^{f, \dagger}$ \\ Ray Bell, ${ }^{\mathrm{g}}$ Dominic Benford, ${ }^{\mathrm{h}}$ Edward Bergin, ${ }^{\mathrm{i}, \uparrow}$ Jeffrey T. Booth, ${ }^{\mathrm{j}}$ \\ Charles M. Bradford ${ }^{\mathrm{j}, \dagger}$ Damon Bradley, ${ }^{\mathrm{a}}$ Sean Carey, ${ }^{\mathrm{d}}$ Ruth Carter, ${ }^{\mathrm{a}}$ \\ Asantha Cooray, ${ }^{\mathrm{k}, \dagger}$ James Corsetti, ${ }^{a}$ Larry Dewell, ${ }^{\mathrm{g}}$ Michael DiPirro, ${ }^{\mathrm{a}}$ \\ Bret G. Drake, Matthew East, ${ }^{\text {b }}$ Kimberly Ennico $\odot{ }^{\text {m, }}{ }^{\dagger}$ Greg Feller, ${ }^{\text {n }}$ \\ Angel Flores, ${ }^{a}$ Jonathan Fortney, ${ }^{o, \dagger}$ Zachary Granger, ${ }^{\mathrm{g}}$ \\ Thomas P. Greene $\odot,{ }^{m}$ Joseph Howard, ${ }^{a}$ Tiffany Kataria, ${ }^{j, \dagger}$ John S. Knight, ${ }^{p}$ \\ Charles Lawrence, ${ }^{\mathbf{j}}$ Paul Lightsey $\odot{ }^{\text {p }}$ John C. Mather $\odot,{ }^{\text {a }}$ \\ Margaret Meixner, ${ }^{\mathbf{q}, \dagger}$ Gary Melnick, ${ }^{\mathbf{r}, \dagger}$ Craig McMurtry $\odot,{ }^{\mathbf{s}}$ \\ Stefanie Milam $\odot,{ }^{a}, \uparrow$ Samuel H. Moseley, ${ }^{\mathrm{t}}$ Desika Narayanan, ${ }^{\mathrm{u}, \dagger}$ \\ Alison Nordt, ${ }^{\mathrm{g}}$ Deborah Padgett, ${ }^{\mathrm{j}, \dagger}$ Klaus Pontoppidan $\odot,{ }^{\mathrm{v}, \dagger}$ \\ Alexandra Pope $\odot,{ }^{\mathrm{w}, \dagger}$ Gerard Rafanelli, ${ }^{\mathrm{x}}$ David C. Redding, ${ }^{\mathrm{j}}$ George Rieke, ${ }^{\mathrm{y}}$ \\ Thomas Roellig, ${ }^{\mathrm{m}, \dagger}$ Itsuki Sakon $\odot{ }^{\mathrm{z}}$ Carly Sandin $\odot,{ }^{\mathrm{a}}$ Karin Sandstrom $\odot,{ }^{\mathrm{A}, \dagger}$ \\ Anita Sengupta, ${ }^{j}$ Kartik Sheth, ${ }^{\mathrm{h}}$ Lawrence M. Sokolsky, ${ }^{\mathrm{g}}$ \\ Johannes Staguhn $\odot,{ }^{a, B, \dagger}$ John Steeves $\odot,{ }^{j}$ Kevin Stevenson $\odot,{ }^{C},{ }^{\dagger}$ Kate Su $\odot,{ }^{\mathbf{y}, \dagger}$ \\ Joaquin Vieira, ${ }^{\mathbf{D}, \dagger}$ Cassandra Webster, ${ }^{\text {a }}$ Martina Wiedner $\odot,{ }^{\mathbf{E}}$ \\ Edward L. Wright, ${ }^{\text {, } \dagger}$ Chi Wu, ${ }^{\text {a }}$ David Yanatsis, ${ }^{b}$ Jonas Zmuidzinas, ${ }^{\text {G }}$ \\ and the Origins Space Telescope Mission Concept Study Team \\ ${ }^{a}$ NASA Goddard Space Flight Center, Sciences and Exploration Directorate, Greenbelt, \\ Maryland, United States \\ ${ }^{b}$ L3Harris Corporation, Rochester, New York, United States \\ ${ }^{\mathrm{c}}$ Northrop-Grumman Space Systems, Redondo Beach, California, United States \\ ${ }^{\mathrm{d}}$ Caltech/Infrared Processing and Analysis Center, Pasadena, California, United States \\ e University of Connecticut, Department of Physics, Storrs, Connecticut, United States \\ ${ }^{\mathrm{f}}$ University of Maryland, Astronomy Department, College Park, Maryland, United States \\ ${ }^{\mathrm{g}}$ Lockheed-Martin Advanced Technology Center, Palo Alto, California, United States \\ ${ }^{\mathrm{h}}$ Astrophysics Division, Science Mission Directorate, NASA Headquarters, Washington, DC, \\ United States \\ ${ }^{\mathrm{i}}$ University of Michigan, Department of Astronomy, Ann Arbor, Michigan, United States \\ ${ }^{\mathrm{j} J e t}$ Propulsion Laboratory, Caltech, Pasadena, California, United States \\ ${ }^{k}$ University of California, Irvine, Department of Physics and Astronomy, Irvine, California, \\ United States \\ ${ }^{1}$ Aerospace Corporation, El Segúndo, California, United States \\ ${ }^{m}$ NASA Ames Research Center, Mountain View, California, United States \\ ${ }^{\mathrm{n}}$ Coherent, Inc., Santa Clara, California, United States \\ 'University of California, Santa Cruz, Astronomy and Astrophysics Department, Santa Cruz, \\ California, United States \\ ${ }^{\mathrm{p}}$ Ball Aerospace Corporation, Boulder, Colorado, United States \\ ${ }^{q}$ Universities Space Research Association, Columbia, Maryland, United States \\ ${ }^{\mathrm{r}}$ Harvard-Smithsonian Center for Astrophysics, Cambridge, Massachusetts, United States \\ ${ }^{\mathrm{s}}$ University of Rochester, Department of Physics and Astronomy, Rochester, New York, \\ United States \\ 'Quantum Circuits, Inc., New Haven, Connecticut, United States \\ university of Florida, Department of Astronomy, Gainesville, Florida, United States \\ ${ }^{\vee}$ Space Telescope Science Institute, Baltimore, Maryland, United States \\ ${ }^{\mathrm{w}}$ University of Massachusetts, Department of Astronomy, Amherst, Massachusetts, \\ United States \\ ${ }^{\mathrm{x}}$ Raytheon Space and Airborne Systems, El Segundo, California, United States
}


${ }^{\mathrm{y}}$ The University of Arizona, Department of Astronomy and Steward Observatory, Tucson, Arizona, United States

${ }^{7}$ The University of Tokyo, School of Science, Tokyo, Japan

${ }^{A}$ University of California, San Diego, CASS, San Diego, California, United States

${ }^{\mathrm{B}}$ The Johns Hopkins University, Department of Physics and Astronomy, Baltimore, Maryland, United States

${ }^{\mathrm{C}}$ The Johns Hopkins University Applied Physics Laboratory, Laurel, Maryland, United States

${ }^{\mathrm{D}}$ University of Illinois, Department of Astronomy, Urbana-Champaign, Urbana, Illinois, United States

${ }^{\mathrm{E}}$ Sorbonne Université, Observatoire de Paris, CNRS, LERMA, Paris, France

${ }^{\mathrm{F}}$ UCLA, Division of Astronomy and Astrophysics, Los Angeles, California, United States

${ }^{\mathrm{G}}$ Caltech, Division of Physics, Mathematics and Astronomy, Pasadena, California, United States

Abstract. The Origins Space Telescope will trace the history of our origins from the time dust and heavy elements permanently altered the cosmic landscape to present-day life. How did galaxies evolve from the earliest galactic systems to those found in the universe today? How do habitable planets form? How common are life-bearing worlds? We describe how Origins was designed to answer these alluring questions. We discuss the key decisions taken by the Origins mission concept study team, the rationale for those choices, and how they led through an exploratory design process to the Origins baseline mission concept. To understand the concept solution space, we studied two distinct mission concepts and descoped the second concept, aiming to maximize science per dollar and hit a self-imposed cost target. We report on the study approach and describe the concept evolution. The resulting baseline design includes a 5.9-m diameter telescope cryocooled to $4.5 \mathrm{~K}$ and equipped with three scientific instruments. The chosen architecture is similar to that of the Spitzer Space Telescope and requires very few deployments after launch. The cryo-thermal system design leverages James Webb Space Telescope technology and experience. (0) The Authors. Published by SPIE under a Creative Commons Attribution 4.0 Unported License. Distribution or reproduction of this work in whole or in part requires full attribution of the original publication, including its DOI. [DOI: 10.1117/1.JATIS.7.1.011014]

Keywords: infrared; space telescope; cryogenic; spectroscopy; mission concept development.

Paper 20074SS received Jun. 16, 2020; accepted for publication Feb. 16, 2021; published online Mar. 13, 2021.

\section{Introduction}

The NASA Astrophysics Roadmap describes the broad outlines of a mission called the FarInfrared Surveyor, which was envisaged as an interferometer, but more fundamentally as a mission that would extend far-IR measurement capabilities in sensitivity, spectroscopy, and angular resolution to regimes far beyond those that had ever been probed before. ${ }^{1}$ Based on science community input, NASA decided in 2015 to sponsor a community-led study of this mission, along with three other "large" missions, in preparation for the 2020 Decadal Survey in Astronomy and Astrophysics. Although large was undefined quantitatively, it was understood that these missions would be comparable in ambition to the Great Observatories: the Hubble Space Telescope, the Compton Gamma Ray Observatory, the Chandra X-Ray Observatory, and the Spitzer Space Telescope. ${ }^{2}$ NASA developed these highly successful missions in response to past Decadal Survey recommendations. The James Webb Space Telescope (JWST), ${ }^{3}$ which is presently undergoing tests in preparation for a fall 2021 launch, and the Nancy Grace Roman Space Telescope, ${ }^{4}$ now in final design and fabrication (phase C), are next in line. Prioritization

\footnotetext{
*Address all correspondence to David Leisawitz, david.t.leisawitz@nasa.gov

${ }^{\dagger}$ Member of the Origins Space Telescope Science and Technology Definition Team

${ }^{\ddagger}$ Members of the study team are listed in the Origins Space Telescope Mission Concept Study Report, which is available at https://asd.gsfc nasa.gov/firs/docs/
} 
of a new large mission by the 2020 Decadal Survey could lead to its development as a successor to the Roman Space Telescope and launch in the 2030s.

For each of the large mission studies, NASA appointed a Headquarters Program Scientist and Deputy Program Scientist, and at a field center a Study Scientist and Study Manager, followed in early 2016 by a Science and Technology Definition Team (STDT). The STDT comprised members of the scientific community whose collective responsibility was to set study priorities. The studies began in December 2015 and concluded with the delivery of final study documents in August 2019.

For the Far-Infrared Surveyor, NASA Headquarters chose the Goddard Space Flight Center to host a Study Office, and thus Goddard was home to the study scientist, the study manager, and most of the study's engineering team. Through Cooperative Agreements, the Study Office engaged industrial partners with relevant expertise at Ball Aerospace, Northrop-Grumman, L3Harris, and Lockheed-Martin. The STDT was expanded to include international (non-voting) members, several of whom served as liaisons to the Canadian Space Agency, the Centre National d'Études Spatiales (CNES), the European Space Agency (ESA), the Japan Aerospace Exploration Agency (JAXA), the Netherlands Institute for Space Research, and the Swedish National Space Agency. The STDT reached out to the wider community and engaged hundreds of scientists around the world in developing potential science use cases and establishing scientific priorities. These use cases were documented, but not published, and some of them were developed into science white papers and submitted to the Decadal Survey. International partners in Japan and a European consortium based in France studied two of the instruments considered for inclusion in the mission. The STDT adopted the name Origins Space Telescope for the mission based on its science goals, and hereafter we will use this name or the abbreviation Origins.

The study team benefited not only from the Astrophysics Roadmap's description of the FarInfrared Surveyor, ${ }^{1}$ but also from past mission studies, ${ }^{5-8}$ experience with JWST, and fifteen farinfrared community workshops convened between 1999 and 2018. Through these workshops, the community voiced an interest in two ambitious missions: a large, cold single-aperture far-IR telescope for maximum sensitivity, and a long-baseline spatio-spectral interferometer for maximum angular resolution. ${ }^{9}$ Because no consensus emerged from the series of workshops as to which mission should go first, the STDT began with a clean slate and recognized the imperative objectively, transparently, and expeditiously to choose between an interferometer and a singleaperture telescope.

The foundational goal for the study was to develop a scientifically compelling, executable mission concept for Origins, subject to the boundary condition that it would be a large infrared space mission. Neither large nor "infrared" was defined ab initio. Furthermore, like beauty, "compelling" science is in the eye of the beholder, and "executability," while it certainly implies technology readiness, technical feasibility, and programmatic relevance, might or might not include cost as a factor. The STDT defined these terms for Origins as the study progressed. We decided to focus on a mission with three main science goals, each with corresponding objectives and traceable to higher-level NASA goals (Fig. 1): How did galaxies evolve from the

\begin{tabular}{|c|c|c|c|}
\hline NASA Goal & How Does the Universe Work? & How Did We Get Here? & Are We Alone? \\
\hline $\begin{array}{l}\text { Origins } \\
\text { Science } \\
\text { Goals }\end{array}$ & $\begin{array}{l}\text { How do galaxies form stars, make heavy } \\
\text { elements, and grow their central super- } \\
\text { massive black holes from reionization } \\
\text { to today? }\end{array}$ & $\begin{array}{l}\text { How do the conditions for } \\
\text { habitability develop during the } \\
\text { process of planet formation? }\end{array}$ & $\begin{array}{l}\text { ng } \\
\text { upport life? }\end{array}$ \\
\hline $\begin{array}{l}\text { Origins } \\
\text { Scientific } \\
\text { Objectives }\end{array}$ & $\begin{array}{l}\text { 1. How does the relative growth of stars and super- } \\
\text { massive black holes in galaxies evolve with time? } \\
\text { 2. How do galaxies make heavy elements, dust, and } \\
\text { organic molecules? } \\
\text { 3. How do the relative energetics from supernovae } \\
\text { and quasars influence the interstellar medium of } \\
\text { galaxies? }\end{array}$ & $\begin{array}{l}\text { 1. What role does water play in the formation } \\
\text { and evolution of habitable planets? } \\
\text { 2. How and when do planets form? } \\
\text { 3. How were water and life's ingredients } \\
\text { delivered to Earth and to exoplanets? }\end{array}$ & $\begin{array}{l}\text { 1. What fraction of terrestrial planets } \\
\text { around M and K-dwarf stars }{ }^{1} \text { has tenu- } \\
\text { ous, clear, or cloudy atmospheres? } \\
\text { 2. What fraction of terrestrial M-dwarf } \\
\text { planets is temperate? } \\
\text { 3. What types of temperate, terrestrial, } \\
\text { M-dwarf planets support life? }\end{array}$ \\
\hline
\end{tabular}

${ }^{1} \mathrm{~K}$-star planet hosts will be included in the initial reconnaissance in case they should turn out to be interesting for followup observations, but the planned biosignature search will focus on M stars.

Fig. 1 Scientific goals and objectives for the Origins Space Telescope. 
earliest galactic systems to those found in the universe today? How do habitable planets form? How common are life-bearing worlds? After initially ignoring cost, we reached consensus on the notion that, to be executable, a mission must be affordable, and we aimed for a mission that would cost approximately \$5B. Finally, based on the established scientific objectives, we decided that Origins would cover the wavelength range from 2.8 to $588 \mu \mathrm{m} .{ }^{10}$ While more information about exoplanet atmospheres can be found at wavelengths shorter than $2.8 \mu \mathrm{m}$, detectable biosignatures exist in the 2.8 to $20 \mu \mathrm{m}$ range, and going shorter would demand better optical system performance and drive up the cost. While valuable information about galaxy evolution lies at wavelengths $>588 \mu \mathrm{m}$, such wavelengths are accessible from the ground.

This paper describes the STDT's key decisions, the rationale for those choices, and how they led through an exploratory design process to the Origins baseline mission concept ${ }^{11}$ which the STDT recommended to the 2020 Decadal Survey in written and oral reports in August and November 2019, respectively. To arrive at the baseline design, we studied two distinct mission concepts and descoped the second one, aiming to maximize science per dollar and hit a selfimposed cost target. The first concept had an open architecture such as JWST, and the second one, an architecture much like that of the Spitzer Space Telescope. Origins baseline design details are summarized in a series of companion papers in this JATIS special section (see Ref. 11 and references therein). Readers who are not already familiar with the Origins baseline mission concept will find it helpful to read papers ${ }^{10,11}$ before reading the rest of this paper. The paper is organized as follows. Section 2 describes the STDT's open study philosophy, the engineering focus on cryo-thermal design, and the overall study approach. Section 3 describes trades and key decisions that led to evolution of the mission concept. Topics reserved for future study are briefly described in Sec. 4. We summarize in Sec. 5.

\section{Origins Study Approach}

\subsection{Community Involvement}

Community involvement was an intentional and important aspect of the Origins mission concept study. As noted above, Origins has roots in the NASA Astrophysics Roadmap, ${ }^{1}$ in which a committee representing the astrophysics community envisions a far-IR Surveyor mission that offers measurement capabilities vastly superior to any prior far-IR mission. The Origins STDT, itself comprising representatives of the community, actively engaged many additional community members in developing and documenting potential science objectives and use cases for the mission. Throughout the study, the STDT's virtual and face-to-face meetings were open and often attended by members of the wider community. Finally, if Origins is prioritized by the 2020 Decadal Survey and developed by NASA, Origins General Observers from the global scientific community will use the telescope to answer the mission-driving science questions and make unexpected, transformative discoveries. ${ }^{10}$

\subsection{Focus on Cryo-Thermal Design}

Based on decades of experience with infrared space telescopes going back to the Infrared Astronomical Satellite in the early 1980s ${ }^{12}$ we knew that striking scientific achievements were made possible by the exceptional sensitivity afforded by cryogenically cooled telescopes. It was clear from the outset that Origins is a cryogenic mission. For this reason, we never diverted our attention from the cryo-thermal system design. ${ }^{13}$ No design decision was taken without understanding its impact on the system's thermal performance. We appointed a highly experienced cryogenic physicist as chief engineer and system architect. We knew from JWST the importance of keeping warm electronics distant and well isolated from cold optical surfaces. We knew from many past infrared missions ${ }^{12,14-19}$ that expendable cryogen is mission lifetime-limiting and consumes mass and volume that could be better used to maximize measurement capabilities if mechanical cryocoolers were employed. We were aware of the state-of-the-art and the potential for advancements in cryocooler technology. ${ }^{20}$ We meticulously tracked parasitic heat loads and budgeted $100 \%$ margin in the heat-lift capacity of the cryocoolers to allow for thermal model

J. Astron. Telesc. Instrum. Syst. $\quad$ 011014-4 Jan-Mar 2021 • Vol. 7(1) 
uncertainties and permit design flexibility. ${ }^{13}$ Knowing that cryogenic cycling during the integration and test (I\&T) phase is time-consuming and therefore expensive, we took advantage of the fact that cycling time is faster when aided by cryocoolers, as seen in JWST experience when its MIRI cryocooler was activated, and proposed a shorter I\&T program than JWST. Despite its shorter overall duration, the Origins I\&T plan enables cryogenic testing at a higher level of assembly. ${ }^{21}$ Finally, we adopted the JWST approach and allowed for primary mirror segment actuation (albeit in only three dimensions: tip, tilt, and piston), and chose thermally conducting materials for mirrors and metering structures ${ }^{22}$ to minimize temperature gradients and ensure optical component and system-level performance compliant with a preliminary optical system error budget. ${ }^{23}$ The telescope will be diffraction limited at $30 \mu \mathrm{m}$. Based on industry experience with the JWST primary mirror segments and the Origins optical error budget, we concluded that the Origins mirror segments will not require time-consuming cryo-null figuring because they will retain shapes within range of their specifications when they are cooled. Optical performance will be verified at cryogenic temperatures at the component (mirror segment) and subassembly (primary mirror wedge engineering test unit) levels.

\subsection{The Origins Study Team's Approach to Mission Design}

If it were feasible to develop a set of mission concepts that fully explore the mission design solution space, it would be possible simply to choose the design that maximizes science return for a targeted cost, or to find the sweet spot where the science per dollar is maximized. However, each design cycle is labor-intensive and takes a considerable amount of time, necessitating a surgical approach. A methodical and practical approach to cost-constrained mission design involves iteration through three design cycles, each one leading to a point design with known technical capabilities and a cost estimate, affording an opportunity to bracket the cost target with the first two cycles, and then interpolate in an effort to maximize science return at a price point. $^{8,24}$

With no NASA-imposed cost cap, the Origins study team adopted a more exploratory approach, which led to the design evolution shown in Fig. 2. The STDT initially set its sights on a far-infrared observatory that might answer a very wide range of interesting science questions. This helped the team to engage the wider community, which was eager to recommend observing programs for a telescope constrained only by physics. The result was mission "Concept 1."

At its fifth face-to-face meeting in June 2017, with most aspects of the initial engineering design completed, the STDT decided to terminate the Concept 1 study and to develop a second

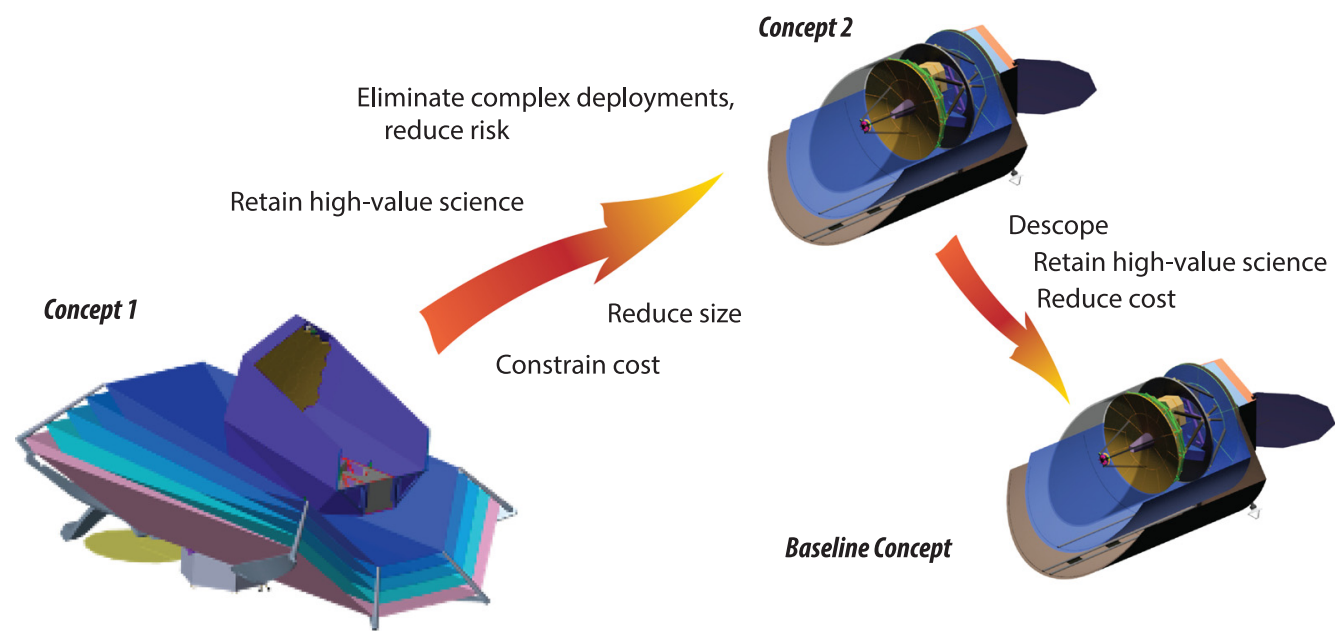

Fig. 2 The Origins study team took an exploratory approach to mission concept development in which two distinct architectures were studied, the second was adopted, and descopes were taken in an effort to develop a concept that maximizes science yield with an executable mission at an affordable cost. 
point design. The study team decided not to allocate resources for detailed cost modeling at this juncture, as it was readily apparent that Concept 1 would be uncomfortably ambitious. Like JWST, Concept 1 required many on-orbit deployments, and JWST's success would not be demonstrated before the Decadal Survey would have to set community priorities. The perceived risk was too great.

At this point in the study, based on the NASA budget thought likely to be available for a large mission after the Roman Space Telescope, the STDT decided to set a \$5B cost target (in fiscal year 2020 dollars) for Origins. Concept 1 would likely have exceeded the newly established cost target by a large but unknown factor, and thus offered little guidance when it came to choosing even the basic system architecture for what would become Concept 2. In August 2017, the Study Office convened a workshop to conceive and evaluate alternative mission architectures. Science return, complexity, and heritage were important figures of merit. Design options were presented to the STDT when it met again face-to-face the following month. The STDT adopted an architecture for Concept 2 that resembles the Spitzer Space Telescope,${ }^{16}$ as shown in Fig. 3; the primary mirror would not be folded for launch and deployed in space, as it was in Concept 1 . The team retained from Concept 1 the science priority to conduct a search for biosignatures in the atmospheres of exoplanets in transiting orbits around nearby late-type dwarf stars, and this drove the requirement for a telescope with at least a 5-m aperture diameter. Later detailed calculations yielded the more precise requirement for a 5.3-m minimum telescope size. (The STDT's prioritized extragalactic study, which requires measurements of a statistically significant sample of galaxies at redshift $z>6$, leads to a similar requirement for a 5-m telescope, albeit with more graceful falloff in scientific capability with decreasing aperture size. ${ }^{11}$ ) Thus, the basic parameters for Concept 2, cost target, architecture, telescope size, and science priorities, ${ }^{10}$ were defined, and the second design cycle could begin.

We designed and analyzed Concept 2 in much greater detail than Concept 1. Knowing that a third full design cycle would not be achievable in the time available to complete the study, and that a high-fidelity cost estimate would only be derivable from a completed design, we used flight system mass as a proxy for cost at $\$ 1 \mathrm{M} / \mathrm{kg}$ (based on analogous missions) throughout the second design cycle, and subtracted 30\% from $\$ 5 \mathrm{~B}$ to allow for cost reserve. We paid close attention to scientific priorities, mass, and descope options during the second design cycle, but Concept 2 still came in heavy, and therefore too expensive in our rough estimation. Thus, we developed a menu of descope options and the estimated cost savings associated with each option.

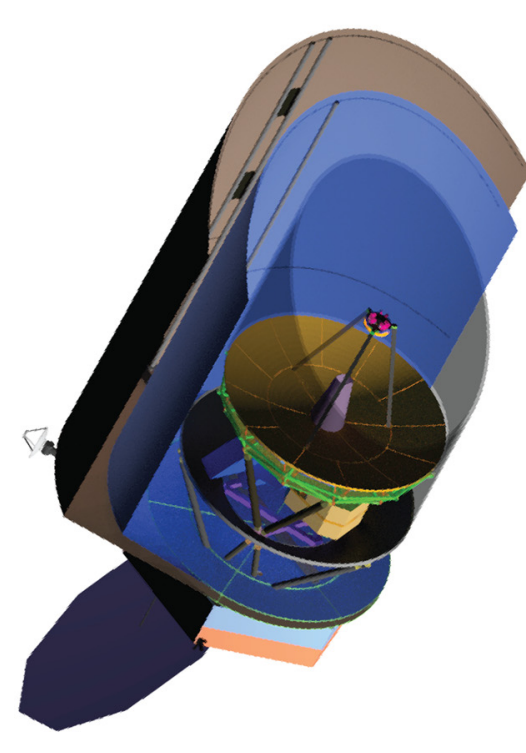

(a)

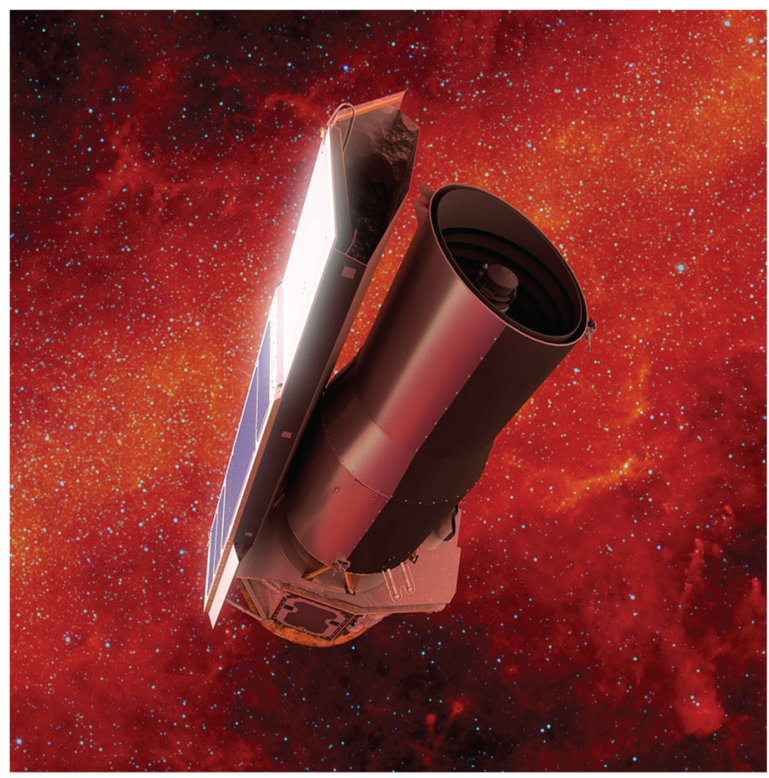

(b)

Fig. 3 (a) Origins Concept 2 compared with the (b) Spitzer Space Telescope (not to scale). Concept 2 has a tubular rather than an open architecture. 
In August 2018, we aimed to maximize science per dollar, made the descopes we felt would be necessary to approach the cost target, based on the mass proxy for cost, and called this design (Concept 2 minus the accepted descopes) the Origins baseline design. Since the baseline design did not benefit from a third design cycle, it is sub-optimal and leaves room for further cost savings. For example, we designed Concept 2 to accommodate four studied instruments, but when one instrument was removed in the descoping process, we made no attempt to reconfigure the space allocated to science instruments. Finally, we used two approaches to derive a high-fidelity estimate of the Origins baseline mission cost. ${ }^{11}$

\section{Origins Concept Evolution Through Trades and Key Decisions}

In this section we compare Concepts 1 and 2 and the Origins baseline mission design and describe the major trades and decisions that led to mission concept evolution. Figure 4 shows the concept evolution schematically, and Fig. 5 summarizes the trades and key decisions.

\subsection{First Decision: Origins is a Single-Aperture Telescope}

The decision process and quantitative analysis that led to selection of a single-aperture telescope are documented in Sec. 3.1.1 of the Origins Space Telescope Interim Report. ${ }^{25}$ This decision was made early in the study (August 2016), based on the STDT's 14 highest-ranked science use cases for the mission out of a total of 46 such cases developed in collaboration with the community. The process unfolded in four steps: (1) the STDT established performance requirements needed to achieve a preliminary set of scientific objectives and separated them into musts and wants; (2) four notional mission concepts were introduced, and approximate performance metrics (angular resolution and sensitivity) were calculated for each concept; (3) the STDT evaluated how well each concept would meet requirements derived from the prioritized use cases, and considered perceived risks associated with each concept; and (4) the STDT chose the best overall concept based on these considerations. The four notional mission concepts were: 5 - and $15-\mathrm{m}$ single-aperture telescopes; a 3.5-m dual-aperture interferometer with up to a 20 -m baseline; and a $6.5-\mathrm{m}$ dual-aperture interferometer with up to a $50-\mathrm{m}$ baseline. Two performance metrics proved to be valuable discriminants. Based on the 14 prioritized use cases, the STDT determined that the mission should provide angular resolution better than $1.24^{\prime \prime}$ at $\lambda=50 \mu \mathrm{m}$ wavelength, and the total set of proposed observations should be completed in under 5 to 10 years integration time, not accounting for observing efficiency. Only the 15-m single-aperture telescope was found to satisfy these conditions. Neither the 5-m telescope nor the two interferometer configurations considered would be able to execute the entire science program in under 10 years. A singleaperture telescope was preferred over a spatial interferometer, in short, because the STDT's favored science required extraordinary sensitivity but not sub-arc sec angular resolution.

\subsection{Mission Concept Evolution}

Figure 6 shows a side-by-side comparison of Concept 1, Concept 2, and the baseline mission concept. We never actually studied a telescope as large as $15 \mathrm{~m}$. Based on earlier studies, we knew that a UV/optical telescope with a primary mirror as large as $9 \mathrm{~m}$ could be folded to fit into a 5-m diameter launch vehicle fairing, so we strove first to find a solution for Origins in that size range.

Concept 1 has a 9.1-m diameter off-axis telescope cryocooled to $4 \mathrm{~K}$ and five science instruments, a medium resolution survey spectrometer (MRSS), a high-resolution spectrometer (HRS), a mid-infrared imager, spectrometer, coronagraph (MISC), a far-infrared imager/polarimeter (FIP), and the Heterodyne Receiver for Origins (HERO). ${ }^{25}$ Collectively, these instruments and this telescope were designed to satisfy the measurement requirements of the fourteen highest-ranked observing programs mentioned in Sec 3.1, and could do a good deal more. The primary mirror, a large Instrument Accommodation Module (IAM), and a five-layer sunshade were stowed for launch and would be deployed on-orbit. Long-wavelength instruments are large, and we learned that the telescope, IAM, sunshield and spacecraft bus would not fit into a 5-m

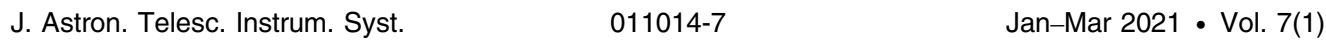




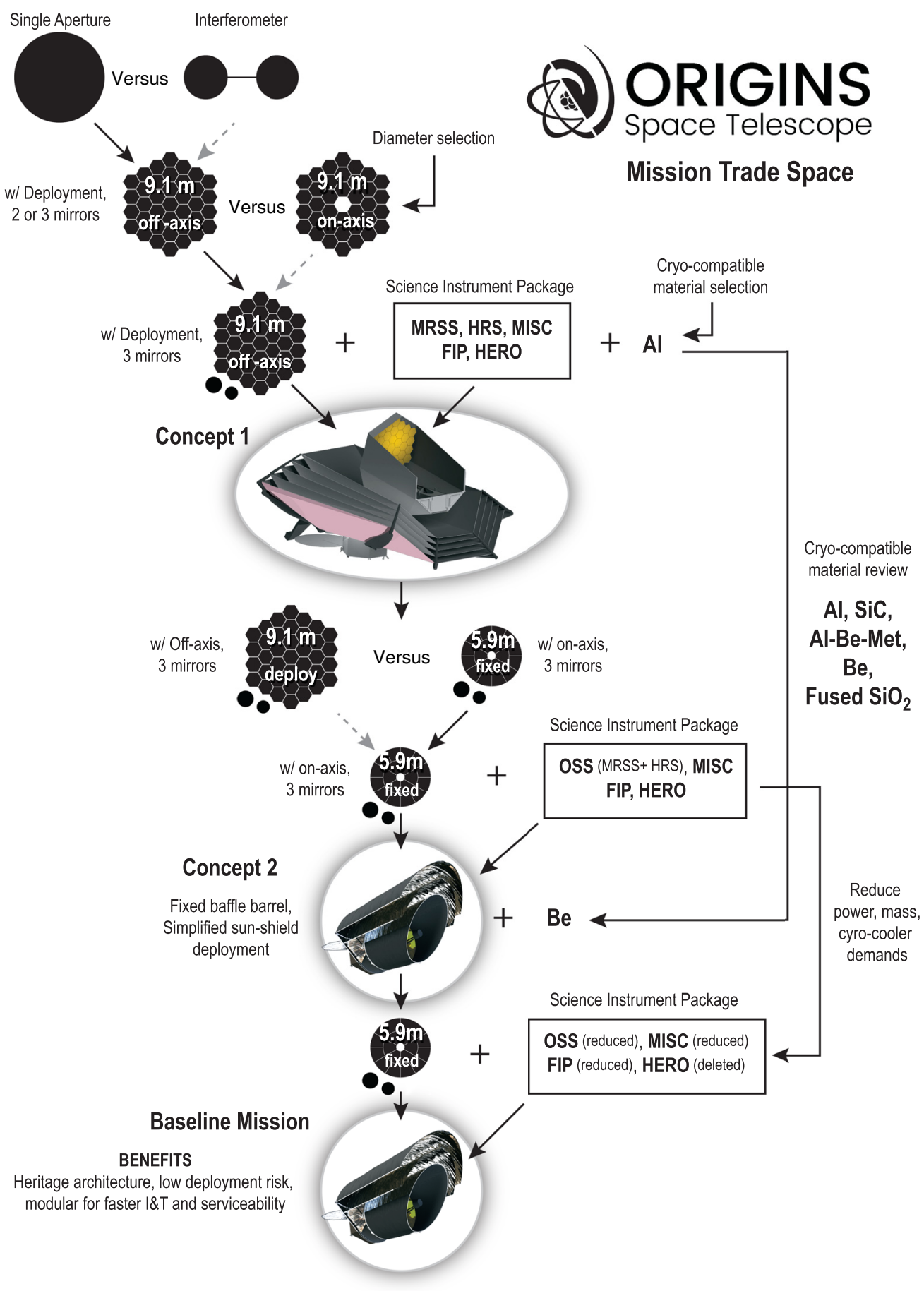

Fig. 4 A single-aperture telescope is best suited to achieve the STDT's prioritized science goals. Subsequent trades look at aperture size, packaging and deployment schemes, materials, and instrument selection. Figure 5 shows the section of this paper in which each trade is discussed.

diameter fairing. Thus, we were forced to adopt NASA's Space Launch System (SLS) with an 8.4-m diameter fairing as the launch vehicle.

Concept 2 has very few and only straightforward deployments, as shown in Fig. 7, and like Concept 1, takes advantage of the large launch vehicles under development for human space exploration (the SLS with an 8.4-m diameter fairing, or a similar commercial vehicle). Study engineers determined that a 5.9-m diameter telescope would leave room in the fairing for a two-layer Spitzer-like sunshield, and the STDT decided to adopt this size, as it would provide 


\begin{tabular}{|c|c|c|c|}
\hline Trade & Decision & Rationale & Discussed in \\
\hline $\begin{array}{l}\text { Single-aperture telescope or interfer- } \\
\text { ometer }\end{array}$ & Single-aperture telescope & $\begin{array}{l}\text { The science case requires superlative spectral line sensitivity and high spectral resolving } \\
\text { power. Science case does not require sub-arcsecond spatial resolution. }\end{array}$ & $\$ 3.1$ \\
\hline $\begin{array}{l}\text { JWST-like (deployable) vs. Spitzer-like } \\
\text { (non- deployable) aperture }\end{array}$ & Spitzer-like & $\begin{array}{l}\text { Minimizes complexity and fits into a launch vehicle that is under development and likely to } \\
\text { exist in the anticipated timeframe. }\end{array}$ & $\S \S 2.3$ and 3.3 .1 \\
\hline $\begin{array}{l}\text { Launch vehicle with } 5 \mathrm{~m} \text { diameter } \\
\text { fairing vs. Iarger vehicle }\end{array}$ & Larger vehicle & $\begin{array}{l}\text { Human exploration and competition are driving development of SLS, Space X BFR, and } \\
\text { Blue Origins' New Glenn. Origins would be volume- constrained in a } 5 \text { m fairing, requiring } \\
\text { complex deployments from a stowed configuration and limiting volume available for } \\
\text { instruments, and on-orbit performance could not be fully-verified with ground tests. }\end{array}$ & $\$ 3.3 .1$ \\
\hline On-axis vs. off-axis telescope & On-axis & $\begin{array}{l}\text { An on-axis three-mirror anastigmat satisfies performance requirements with good Strehl } \\
\text { ratio over a large field-of-view, and enables a larger telescope to fit into the launch vehicle } \\
\text { without necessitating deployment of the secondary mirror. }\end{array}$ & $\$ 3.3 .2$ \\
\hline $\begin{array}{l}\text { Telescope temperature } 4.5 \mathrm{Kvs} \text {. } \\
\text { warmer }\end{array}$ & $4.5 \mathrm{~K}$ & $\begin{array}{l}\text { To satisfy science-driven sensitivity requirements in the far-IR, } 4.5 \mathrm{~K} \text { is preferred, but up to } \\
6 \mathrm{~K} \text { is acceptable. The former can easily be attained with current state of the art cryocooler } \\
\text { technology. A telescope warmer than } 6 \mathrm{~K} \text { would not meet sensitivity requirements. }\end{array}$ & $\$ 3.3 .3$ \\
\hline $\begin{array}{l}\text { Expendable cryogen vs. mechanical } \\
\text { cryocoolers }\end{array}$ & Cryocoolers & $\begin{array}{l}\text { Expendable cryogen would require a large, massive cryostat and would be mission life- } \\
\text { time-limiting, whereas cryocooler technology has matured and has none of these issues. }\end{array}$ & $\$ 3.3 .3$ \\
\hline $\begin{array}{l}\text { Materials: beryllium vs. aluminum vs. } \\
\text { Sic, carbon fiber, or other }\end{array}$ & $\begin{array}{l}\text { Beryllium was chosen for } \\
\text { optical components and } \\
\text { instrument structure }\end{array}$ & $\begin{array}{l}\text { Material properties (e.g., low density, high stiffness and strength, low CTE, high thermal } \\
\text { conductivity) of Be at cryogenic temperatures are superior to alternatives, outweighing } \\
\text { handling challenges. }\end{array}$ & $\$ 3.3 .4$ \\
\hline $\begin{array}{l}\text { Primary mirror segmentation: hex- } \\
\text { agonal vs. keystone-shaped wedges; } \\
\text { various segment sizes and numbers }\end{array}$ & $\begin{array}{l}\text { Circular mirror with key- } \\
\text { stone-shaped segments in } \\
\text { two annuli }\end{array}$ & $\begin{array}{l}\text { Circular maximizes collecting area in the fairing without requiring mirror deployment. Of } \\
\text { four segmentation schemes considered, two are preferred because the segments can be } \\
\text { fabricated in existing facilities and have only two distinct sizes. }\end{array}$ & $\$ 3.3 .5$ \\
\hline $\begin{array}{l}\text { Far-IR detectors: Transition Edge } \\
\text { Sensors (TES) bolometers, Microwave } \\
\text { Kinetic Inductance Detectors (MKIDs), } \\
\text { or another technology }\end{array}$ & $\begin{array}{l}\text { Transition-Edge Sensor } \\
\text { bolometers }\end{array}$ & $\begin{array}{l}\text { TES bolometers and KID detectors are both viable options, and more mature than alterna- } \\
\text { tive technologies. TES bolometers were selected because their room temperature readout } \\
\text { system encompasses requirements for readout of alternative detector types, including KIDs. }\end{array}$ & $\$ 3.3 .6$ \\
\hline Orbit & $\begin{array}{l}\text { Quasi-halo/Lissajous hybrid } \\
\text { at Sun-Earth L2 (SEL2) }\end{array}$ & $\begin{array}{l}\text { SEL2 is far enough from Earth for low background, but dose enough for high-speed data } \\
\text { downlink. The quasi-halo/Lissajous orbit is fuel efficient. }\end{array}$ & $\$ 3.3 .6$ \\
\hline
\end{tabular}

Fig. 5 Origins Space Telescope major trades and decisions.

"science margin" over the 5.3-m minimum required aperture diameter mentioned in Sec. 2.3. The STDT settled on three main science goals for the mission, and for each goal established three well-defined scientific objectives (Fig. 1). ${ }^{10}$ Working again in consultation with the community, the STDT developed 25 use cases for a notional $5.9 \mathrm{~m}, 4 \mathrm{~K}$ telescope, nine in support of the primary objectives and others that were deemed meritorious. To maximize the mission's scientific potential, all of these use cases were allowed to influence instrument choices for Concept 2 , and the measurement requirements flowing from the nine primary objectives were treated as fundamental design drivers. We extended the short wavelength cutoff to 2.8 from $5 \mu \mathrm{m}$ to capture an important methane band at $3.3 \mu \mathrm{m}$ in support of the search for biosignatures. The study team developed a new instrument for Concept 2, the Origins Spectrometer for Surveys (OSS), ${ }^{26}$ which combined MRSS and HRS far-IR spectroscopic capabilities, and we truncated the long wavelength cutoff at $588 \mu \mathrm{m}$ because the atmosphere is relatively transparent at longer wavelengths, enabling complementary measurements with ground-based sub-millimeter and millimeter-wave telescopes. The MISC, ${ }^{27}$ FIP, $^{28}$ and HERO $^{29}$ instrument designs were further developed. Based on its mass and the fiducial $\$ 1 \mathrm{M} / \mathrm{kg}$ cost factor, the total cost of Concept 2 was estimated to be roughly $\$ 8 \mathrm{~B}$, indicating that we should seek $\sim \$ 3 \mathrm{~B}$ in cost savings to reach the target cost.

The team then considered 32 descope options and strove to maximize science per dollar in a less expensive mission, which was to become the Origins baseline concept. The STDT considered but rejected a reduction in telescope size; the baseline telescope is still $5.9 \mathrm{~m}$. The MISC Transit (MISC-T) spectrometer retained all three of its spectral channels, and it offers continuous spectral coverage from 2.8 to $20 \mu \mathrm{m}$, an excellent wavelength range in which to search for biosignatures. However, we removed the MISC camera, which was designed to provide wide-field imaging and low-resolution spectroscopic capability with filters and grisms in the 5 to $28 \mu \mathrm{m}$ range. This change leaves a small gap in Origins' wavelength coverage from 20 to $25 \mu \mathrm{m}$. All six wavelength bands of OSS were retained, giving this instrument continuous spectral coverage from 25 to $588 \mu \mathrm{m}$, an ideal range in which to measure the physical properties of distant galaxies and look for water in protoplanetary disks. OSS keeps its high and ultra-high spectral resolution modes. However, the number of detector pixels was halved, resulting in a factor of two reduction in mapping speed. Only two of FIP's original four spectral channels were retained: the 50 and $250 \mu \mathrm{m}$ bands, and FIP can still make polarimetric measurements. The FIP pixel count was also reduced. The telescope temperature was adjusted from 4 to $4.5 \mathrm{~K}$ to take advantage of a stage common to existing cryocoolers. ${ }^{20}$ In the descope, we gave up the MISC camera, the 100 and $500 \mu \mathrm{m}$ channels of FIP, and the HERO instrument. Even with their reduced pixel counts, OSS 


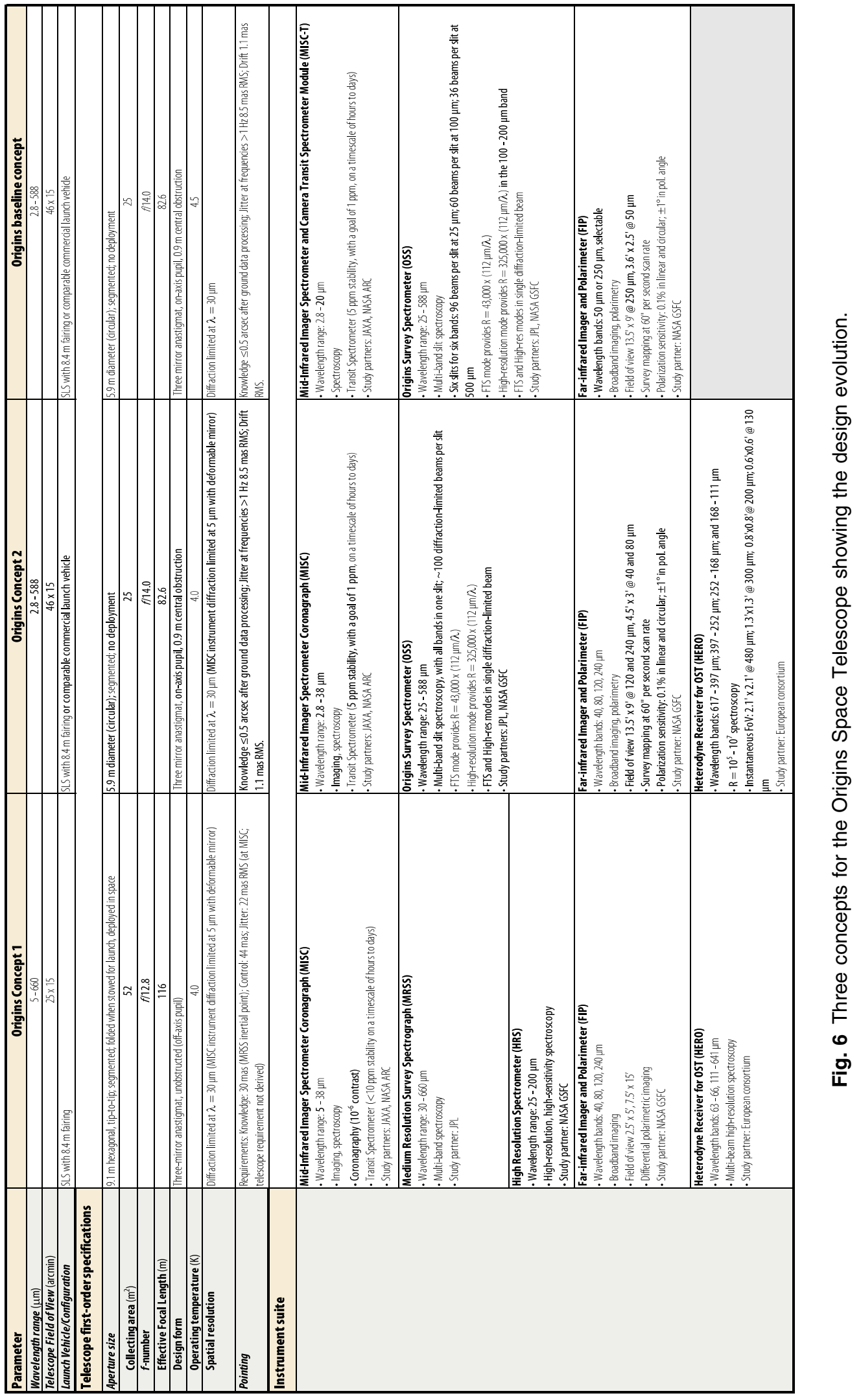




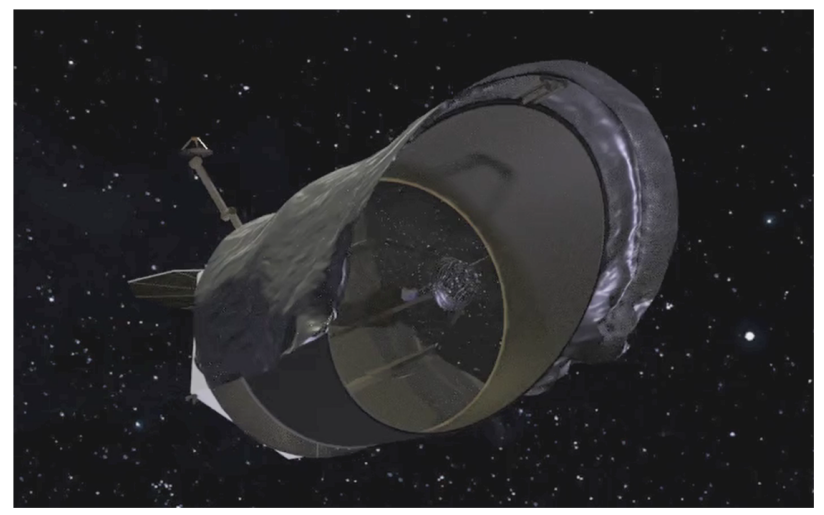

Fig. 7 On-orbit deployment of the Origins Space Telescope. This deployment scheme was adopted for Concept 2 and the baseline design. Origins relies on three simple and standard deployments for its communication antenna, solar array and protective cover ejection, and only one new but simple approach to deploying a two-layer sun shade. Much like a "pop-up tent," the shade is spring loaded and unfurls, then translates to separate the layers. For comparison, to fit into a 5-m fairing, JWST relies on 22 deployment events. (Video 1, MP4, 8 MB [URL: https://doi .org/10.1117/1.JATIS.JATIS.7.1.011014.1]).

and FIP offer spatial-spectral and photometric survey speeds, respectively, more than six orders of magnitude faster than Herschel, and several orders of magnitude faster than JWST and ALMA, which were not designed for wide-area surveys (see Fig. 11 in Ref. 11). By far the greatest cost savings, summing to an estimated $\$ 1.7 \mathrm{~B}$, came from the elimination of the MISC camera and HERO. The tabulated savings account for estimated system-level cost impacts, which go beyond the hardware costs of the instruments themselves. The sacrificed capabilities are visible in the Origins focal plane (Fig. 8). In total, the accepted descopes are expected to reduce the mission cost by $\sim \$ 2 \mathrm{~B}$. The STDT estimated qualitatively that, with these changes, the Origins baseline mission would still be able to accomplish $80 \%$ of the science represented by the 25 use cases adopted for Concept 2, without significantly compromising the nine primary scientific objectives shown in Fig. 1. When carefully modeled, the baseline mission cost ( $\$ 6.7$ to $7.3 \mathrm{~B}$ at the $50 \%$ and $70 \%$ confidence levels, respectively) ${ }^{11}$ turned out to be greater than but in reasonable agreement with $\$ 8 \mathrm{~B}$ to $\$ 2 \mathrm{~B}$, considering that $\$ 8 \mathrm{~B}$ and $\$ 2 \mathrm{~B}$ were rough estimates.

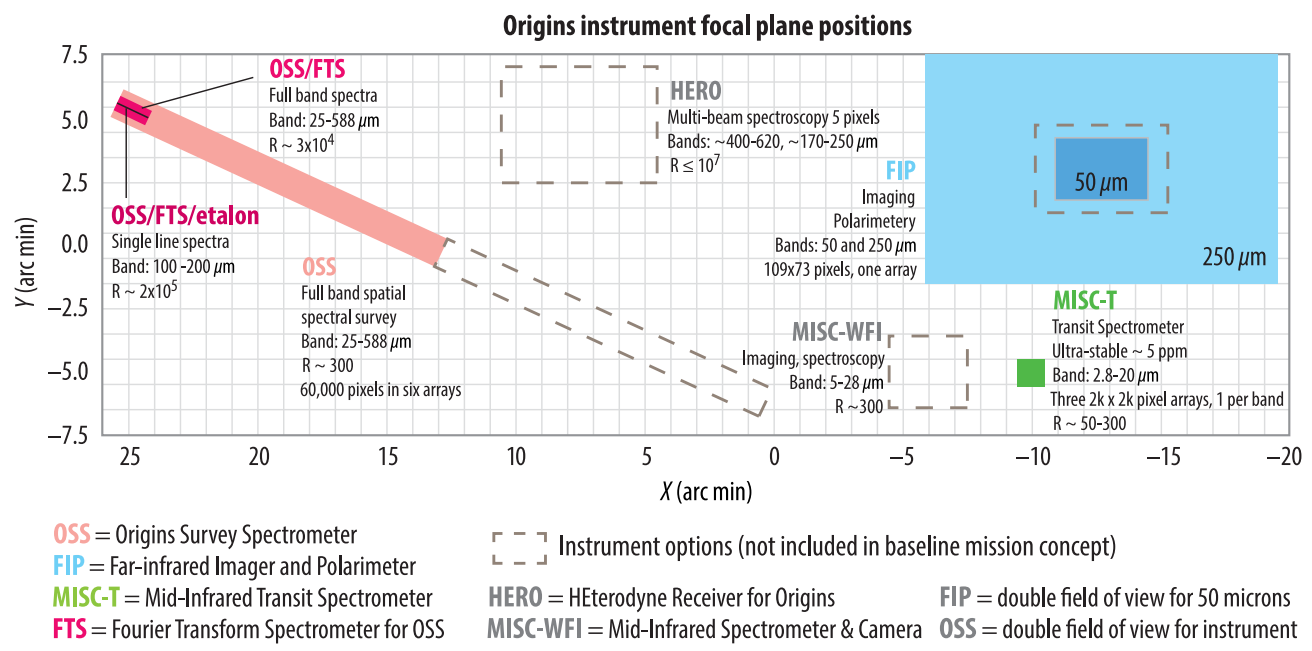

Fig. 8 Concept 2 instrument footprints in the Origins focal plane. Items with a dashed line border were descoped to derive the baseline mission concept. 


\subsection{Trades and Decisions}

Figure 5 summarizes the major trades and decisions reached during the Origins mission concept study. Experience with Spitzer, ${ }^{16}$ the Herschel Space Observatory, ${ }^{19}$ and JWST, ${ }^{3}$ and Concept 1 , strongly influenced our decisions.

\subsubsection{Launch vehicle and mission architecture}

We initially considered a launch vehicle with a 5-m diameter fairing, similar to vehicles that exist now. JWST suggests that this would be possible, ${ }^{30}$ but that it would require a complex deployment scheme. Indeed, Goddard studies of folded configurations for a UV/optical telescope indicated that a telescope as large as $9 \mathrm{~m}$ could be accommodated in a 5-m fairing. However, the Concept 1 design cycle enabled us to gauge the sizes of the Origins instruments, and we found that the observatory was too large for a 5-m fairing. The STDT's science goals for Concept 2 demanded a telescope with light collecting area close to that of JWST, $25 \mathrm{~m}^{2}$. We reconsidered smaller vehicles when downsizing from Concept 1 to Concept 2 but decided, after consulting with many experts, that the benefits of a lower-risk design-one that does not require deployment of the primary mirror-outweighed dependence on launch vehicles still in development, especially given that three suitable vehicles are advancing in parallel, driven by human exploration goals.

\subsubsection{Off-axis versus on-axis optical configuration}

In an off-axis optical design, the primary mirror is unobstructed by the secondary or its supporting structure, and this design form has two advantages: a cleaner point spread function with lower sidelobes, and more light-collecting area for a given primary mirror diameter. On the other hand, such a configuration is less compact than an on-axis design. We opted for an off-axis configuration in Concept 1 and found that it would fit into an 8.4-m fairing on NASA's SLS or a similar launch vehicle. ${ }^{25}$ However, to satisfy the light-collecting area requirement in a Spitzer-like architecture while minimizing deployments, we chose an on-axis three-mirror anastigmat for the optical design in Concept 2. We took advantage of the fact that beam irregularities would have minimal adverse impact on a mission whose scientific objectives would be fulfilled primarily through spectroscopy, rather than imaging.

\subsubsection{Operating temperature}

To suppress the observatory's thermal self-emission and approach the natural astrophysical background photon noise level-essential to achieving Origins' science goals- the telescope's optical elements must be cooled to $<6 \mathrm{~K} .{ }^{11}$ At wavelengths $>200 \mu \mathrm{m}$, the background noise level is extremely sensitive to telescope temperature: $4 \mathrm{~K}$ is much better than $6 \mathrm{~K}$. Past far-infrared missions, including Spitzer, were cooled with expendable cryogens, but that approach requires a large, massive cryostat and limits the life of the mission. Advances in cryocooler technology for JWST, Hitomi, and other missions enabled us to adopt mechanical cryocoolers rather than expendable cryogens for Origins. ${ }^{13}$ As shown in Fig. 6, we adopted $4 \mathrm{~K}$ as the operating temperature in Concept 1, but later relaxed that temperature to $4.5 \mathrm{~K}$ to take advantage of current-stateof-the-art cryocooler technology. ${ }^{13}$ The quoted operating temperature is the temperature of the telescope, including the baffle, mirrors, and support structure, and the volume that houses the instruments.

\subsubsection{Materials}

High stiffness, low density, high thermal conductivity, and low coefficient of thermal expansion (CTE) are critical material properties for Origins. Thermal performance is more important than mass in consideration of the primary mirror, leading to our adoption of the same material for the mirror and its backplane, and other cold-side structures. Beryllium 0 to 30 has excellent specific stiffness and adequate thermal performance, and it has the most relevant cryogenic spaceflight 
heritage. However, beryllium is somewhat brittle, and its dust is toxic, so it requires special care in design (sharp corners and proper clearance holes) and fabrication. We considered a variety of alternative materials, including fused silica, silicon carbide ( $\mathrm{SiC}$ ), aluminum 6061, and an aluminum-beryllium composite, and found that they all have advantages and disadvantages. ${ }^{22} \mathrm{SiC}$, with its high specific stiffness, low strain and low CTE at $4.5 \mathrm{~K}$, and heritage in the Herschel primary mirror, is an attractive alternative to beryllium, but $\mathrm{SiC}$ is also brittle and requires trades on design and processing. Further assessments to address face-sheet thickness, rib thickness, and mirror-segment size are needed for both $\mathrm{Be}$ and $\mathrm{SiC}$. We chose beryllium but consider this choice tentative and subject to further study. The ultimate choice will be influenced by new gap-filling property measurements at cryogenic temperatures. Composite materials can be used for structures to save mass wherever possible (i.e, where good thermal performance and CTE matching are not important).

\subsubsection{Primary mirror segmentation}

Existing facilities would not support the fabrication of a monolithic mirror as large as $5.9 \mathrm{~m}$, so we assumed from the outset that the Origins primary mirror would be segmented. In Concept 1 , the primary mirror was comprised of 37 hexagonal segments, each $1.277-\mathrm{m}$ flat-to-flat, and folded around the instrument module (IAM) for launch. ${ }^{25}$ When we turned our attention to Concept 2 and the baseline concept, to maximize light-collecting area in the cylindrical launch vehicle fairing, we adopted a round mirror comprised of keystone-shaped segments. In consultation with our industrial partners, we studied four segmentation schemes, found two of them compatible with existing fabrication capabilities, and adopted a scheme consisting of an inner ring of six segments and radial width $1.200 \mathrm{~m}$, and an outer annulus of 12 segments and radial width $1.294 \mathrm{~m}$. Within each annulus the mirror shapes are identical, except that the inner-ring segments are notched in opposite corners to allow feed-through of the secondary mirror support structure.

\subsubsection{Far-infrared detectors}

For several reasons, we adopted TES bolometers as far-IR detectors in the Origins baseline mission concept, but we consider this choice tentative. First, both TES bolometers and MKIDs are approaching the Origins detector sensitivity and pixel count requirements, and more advanced overall than alternative detector types. Second, because the warm readout system for TES bolometers needs additional signals and electronics beyond those needed for MKIDs, we chose TES bolometers to bound the design. Most importantly, we plan to select a single detector type for the Origins far-infrared instruments after further technology maturation. Thus, we wanted to study a mission point design with a single far-IR detector type. In summary, we chose TES bolometers because they require more development in their warm readout electronic system than MKIDs, and our aim was to envelope the interesting solution space.

\subsubsection{Orbit}

Origins must be far enough from Earth to facilitate cooling and provide a relatively unobstructed view of the sky, yet near enough to enable high-speed data downlink. These requirements favor an orbit around the Sun-Earth Lagrange 2 (SEL2) point. The observatory also must avoid shadows from the Earth and the Moon. When the orbit is further constrained to ensure that no stray light from the Moon reaches the inside of the primary mirror baffle, and optimized to minimize fuel consumption, our orbital dynamics experts recommended a quasi-halo/Lissajous hybrid orbit with a six-month period around SEL2.

\subsubsection{Mission lifetime}

We estimate that the community will be able to achieve the goals and objectives given in Fig. 1 in two years with the Origins baseline mission, ${ }^{10}$ but a competitive general observing program will extend our scientific horizon to places not yet even imagined. To enable such discovery science

J. Astron. Telesc. Instrum. Syst. $\quad$ 011014-13 Jan-Mar 2021 • Vol. 7(1) 
Origins will have a minimum five-year lifetime, a 10-year design lifetime, and an option for servicing that could replenish the propellant supply and replace instruments to extend its lifetime beyond 10 years.

\section{Future Origins Study Topics}

Our pre-decadal study addressed a number of open questions and converged on a scientifically powerful, low-risk, and executable mission concept, yet a great deal of additional preparatory work is needed to bring the mission concept to a successful Preliminary Design Review (PDR), nominally in November 2028. If the decadal survey recommends Origins, the mission will enter pre-phase A, during which open questions will be brought to closure and the mission concept will be refined and optimized. Ultimately, the mission that flies will likely resemble the baseline mission concept, ${ }^{11}$ but a cold JWST concept ${ }^{30}$ will receive further consideration, as it would leverage existing ground support infrastructure and a proven design, once JWST is launched and commissioned. Even if the Spitzer-like architecture is adopted, design details can be expected to change in response to future studies.

The Origins Space Telescope Technology Development Plan ${ }^{31}$ presents a roadmap to mature the Origins-enabling technologies (also see Ref. 32 for a contemporary review of far-infrared mission-enabling and enhancing technologies). According to the plan, during phase $\mathrm{A}$, all of the technologies will substantially progress toward their technology readiness level (TRL) 6 milestones. Detector and cryocooler technology maturation will be closely monitored, and choices will be made based on the availability of TRL- 6 technology in time for the mission PDR. (NASA is developing innovative approaches to project management as these words are written, and future large missions may have to reach their TRL 6 milestone earlier. The Origins mission schedule would slip by about a year if the technologies were required to reach TRL 6 in time for the instrument PDRs.)

A future Origins science team will conduct additional analyses to estimate definitively the precision to which spectral line strengths and continuum fluxes can be derived from spatially confused observations of distant galaxies with the planned Origins spectroscopic measurement capabilities. A future engineering team will optimize the Origins design to accommodate the instruments, and the instruments will be packaged more compactly and make better use of the central sweet spot in the telescope's field of view (Fig. 8), where the Strehl ratio approaches 1. A high-fidelity thermal model will be used and validated through ground testing to ensure an accurate understanding of the observatory's thermal performance and enable future design trades. For example, the thermal performance will be sensitive to the location of the solar array. Optical communication systems are developing rapidly today and will be revisited to ensure they meet the Origins requirements for data downlink. While some material properties are currently unknown at $\sim 4 \mathrm{~K}$, new measurements will enable new material trades and good choices for the flight system's optical and structural elements and sunshields. ${ }^{22}$ Many additional study topics are common to all large missions in formulation and do not warrant discussion here.

\section{Summary}

We have described the key decisions taken by the Origins mission concept study team, the rationale for those choices, and how they led through an exploratory design process to the Origins baseline mission concept presented to the 2020 Decadal Survey in Astronomy and Astrophysics. Most importantly, we decided to take advantage of the large launch vehicles being developed for NASA's human exploration program and adopted an architecture like that of the Spitzer Space Telescope, motivated by its proven thermal performance and minimal dependence on on-orbit deployments. The cryo-thermal system design leverages JWST technology and experience. We studied two distinct mission concepts and descoped the second concept, aiming to hit a \$5B selfimposed cost target. The resulting baseline design includes a 5.9-m diameter telescope cryocooled to $4.5 \mathrm{~K}$ and equipped with three scientific instruments, which cover the entire wavelength range 2.8 to $588 \mu \mathrm{m}$, except for a small gap from 20 to $25 \mu \mathrm{m}$. The Origins baseline mission concept is executable and, with margin, capable of definitively addressing the scientific 
objectives given in Fig. 1, and three compelling science goals: (1) How do galaxies form stars, make heavy elements, and grow their central supermassive black holes throughout the history of the universe?; (2) How do the conditions for habitability develop during the process of planet formation?; and (3) Do planets orbiting M-dwarf stars support life?

\section{Acknowledgments}

We took excerpts from the SPIE Proceedings paper "The Origins Space Telescope: mission concept overview," in Proc. SPIE 10698-40 (2018) and from the Origins Space Telescope Mission Concept Study Final Report. The authors are grateful to the many institutions that sponsored and contributed to the successful Origins Space Telescope mission concept study. To enable the community to prepare for the 2020 Decadal Survey, the National Aeronautics and Space Administration (NASA) sponsored studies of four large mission concepts, of which Origins was one. We thank NASA, for funding these studies. NASA's Goddard Space Flight Center (GSFC) contributed substantial additional labor support, which enabled us to explore options, make wellinformed engineering decisions, and develop an executable mission concept. The Japan Aerospace Exploration Agency, JAXA, the Canadian Space Agency, CSA, and a CNES-led European consortium actively participated in the study, with each contributing an instrument design and enabling their team members' travel to study team meetings and concurrent engineering sessions. Domestic study participants included many academic institutions, several NASA centers (Ames Research Center and Marshall Space Flight Center, as well as GSFC), the Caltech Jet Propulsion Laboratory, and industry (Ball Aerospace, Northrop-Grumman, Lockheed-Martin, and L3 Harris), as reflected in the authors' affiliations. The authors wish to express their deep gratitude to the hundreds of community members worldwide who contributed to the Origins mission concept study by sharing their thoughts on science priorities, reviewing the science case and engineering designs, developing graphics, formatting reports, taking notes, managing study resources, and making travel arrangements. It took a village. Last, but not least, we thank three JATIS reviewers, whose feedback on the original manuscript led to substantial improvements.

\section{References}

1. C. Kouveliotou et al., "Enduring quests, daring visions-NASA astrophysics in the next three decades," 2013, https://science.nasa.gov/science-committee/subcommittees/nac-astrophysicssubcommittee/astrophysics-roadmap.

2. M. Harwit and V. Neal, "The great observatories for space astrophysics," NASA-CR-176754 (1986).

3. J. Gardner et al., "The James Webb space telescope," Space Sci. Rev. 123, 485 (2006).

4. "Nancy Grace Roman Space Telescope," https://roman.gsfc.nasa.gov (accessed 11 December 2020).

5. M. W. Werner and T. B. H. Kuiper, "A large deployable reflector for infrared and submillimeter astronomy from space," in Proc. 2nd ESO Infrared Workshop, held in Garching, April 20-23, 1982, Garching: European Southern Observatory (ESO), K. Kjaer and A. F. M. Moorwood, Eds., p. 421 (1982).

6. D. Lester et al., "The single aperture far infrared observatory," Chapter 8 in NASA Space Science Vision Missions, M. S. Allen, Ed., pp. 263-300, AIAA (2008).

7. M. Harwit et al., "A kilometer-baseline far-infrared/submillimeter interferometer in space," Chapter 9 in NASA Space Science Vision Missions, M. S. Allen, Ed., pp. 301-326, AIAA (2008).

8. D. Leisawitz et al., "The Space Infrared Interferometric Telescope (SPIRIT): high-resolution imaging and spectroscopy in the far-infrared," Adv. Space Res., 40, 689 (2007).

9. M. Harwit et al., "Far-infrared/submillimeter astronomy from space: tracking an evolving Universe and the emergence of life. A community plan for the far-infrared," 2008, https://old .ipac.caltech.edu/pdf/FIR-SMM_Crosscutting_Whitepaper.pdf.

10. M. Meixner et al., "Origins Space Telescope Science to Design Traceability," J. Astron. Telesc. Instrum. Syst. 7(1), 011012 (2021). 
11. D. Leisawitz et al., "The Origins Space Telescope: baseline mission concept," J. Astron. Telesc. Instrum. Syst. 7(1), 011002 (2021).

12. G. Neugebauer et al., "The Infrared Astronomical Satellite (IRAS) mission," APJ Lett. 278, L1-L6 (1984).

13. M. DiPirro et al., "Origins Space Telescope cryo-thermal system," J. Astron. Telesc. Instrum. Syst. 7(1), 011009 (2021).

14. N. W. Boggess et al., "The COBE mission-Its design and performance two years after launch," Astrophys J. 397, 420 (1992).

15. M. F. Kessler et al., "The Infrared Space Observatory (ISO) mission," Astron. Astrophys. 315, L27-L31 (1996).

16. M. W. Werner et al., "The Spitzer Space Telescope mission," ApJ Suppl. 154, 1-9 (2004).

17. H. Murakami et al., "The Infrared Astronomical Mission AKARI," PASJ 59, 369 (2007).

18. E. L. Wright et al., "The Wide-field Infrared Survey Explorer (WISE): mission description and initial on-orbit performance," Astrophys J. 140, 1868 (2010).

19. G. L. Pilbratt et al, "Herschel Space Observatory. An ESA facility for far-infrared and submillimetre astronomy," Astron. Astrophys. 518, L1-L6 (2010).

20. M. DiPirro et al., "Cryocooling technologies for the Origins Space Telescope," J. Astron. Telesc. Instrum. Syst. 7(1), 011008 (2021).

21. S. Petro et al., "Origins Space Telescope Integration and Testing," J. Astron. Telesc. Instrum. Syst. 6(4), 041502 (2020).

22. C. Sandin et al., "Materials evaluation for the Origins Space Telescope," J. Astron. Telesc. Instrum. Syst. 7(1) (2021).

23. J. Corsetti et al., "Optical design of the Origins Space Telescope," J. Astron. Telesc. Instrum. Syst. 7(1), 011010 (2021).

24. D. A. DiPietro, "A systems engineering approach to architecture development," in INCOSE Int. Symp., Vol. 25, p. 619 (2015).

25. Origins Space Telescope Mission Concept Study Team, “Origins Space Telescope Interim Study Report,” 2018, https://asd.gsfc.nasa.gov/firs/docs/.

26. C. M. Bradford et al., "The Origins Survey Spectrometer (OSS): revealing the hearts of distant galaxies and forming planetary systems with ultrasensitive far-IR spectroscopy," J. Astron. Telesc. Instrum. Syst. 7(1) (2021).

27. I. Sakon et al., "The Origins Mid-Infrared Spectrometer Camera (MISC) baseline and upscope," J. Astron. Telesc. Instrum. Syst. 7(1) (2021).

28. J. Staguhn et al., "The Far-infrared Imager and Polarimeter (FIP) for the Origins Space Telescope,' J. Astron. Telesc. Instrum. Syst. 7(1) (2021).

29. M. Wiedner et al., "HEterodyne Receiver for Origins (HERO)," J. Astron. Telesc. Instrum. Syst. 7(1), 011007 (2021).

30. J. Arenberg et al., "An alternate architecture for the Origins Space Telescope," J. Astron. Telesc. Instrum. Syst. 7(1), 011006 (2021).

31. Origins Space Telescope Mission Concept Study Team, "Origins Space Telescope technology development plan," 2019, https://asd.gsfc.nasa.gov/firs/docs/.

32. D. Farrah et al., "Review: far-infrared instrumentation and technological development for the next decade," J. Astron. Telesc. Instrum. Syst. 5(2), 020901 (2019).

David Leisawitz is NASA study scientist for the Origins Space Telescope and is most interested scientifically in the development of habitable conditions during planet formation. He was PI on the Space Infrared Interferometric Telescope mission concept study and served as NASA Goddard study lead for the Submillimeter Probe of the Evolution of Cosmic Structure. He was mission scientist for the Wide-field Infrared Survey Explorer and deputy project scientist for the Cosmic Background Explorer.

Edward Amatucci has developed everything from robotic devices to scientific instruments for space missions over the past 38 years as an engineer, and currently an instrument systems engineer as a contractor for NASA Goddard Space Flight Center. He earned a bachelor of science in mechanical engineering from the University of Maryland, a master of engineering 
in mechanical engineering and an master of science in technical management from Johns Hopkins University.

Jonathan Arenberg is the chief engineer for Space Science Missions at Northrop Grumman Space Systems. He has worked on the Chandra X-ray Observatory, the Starshade, James Webb Space Telescope, technology development and numerous mission studies, including Origins. His work experience also includes high energy lasers and other optical technologies. He is the author of over 200 conference presentations, papers, a book and book chapters, holds 13 patents and is an SPIE Fellow.

Cara Battersby is an assistant professor of physics at the University of Connecticut. She received her PhD from the University of Colorado at Boulder, then held Submillimeter Array and National Science Foundation Postdoctoral Fellowships at the Harvard Smithsonian Center for Astrophysics. She specializes in star formation in our galaxy's center. Her research group combines observational surveys with numerical simulations to understand the complex interplay of physics involved in star formation in this extreme environment.

Ray Bell is a consultant. He is a retired senior fellow and chief scientist of the Lockheed Martin Advanced Technology Center. He began a long career in astrophysics living and working at the Cosmic Ray Observatory on Mt. Lemmon in southern Arizona and has participated in many space- and ground-based missions for NASA and OGAs.

Jeffrey T. Booth is the program manager of the JPL Astronomy \& Physics Formulation Program Office. He has 20 years of experience at JPL with increasing management responsibilities ranging from group supervisor in the systems engineering section to manager of Competed Missions development to his current role. His expertise includes managing and developing astrophysics, heliophysics, and other space-based scientific missions.

Charles M. Bradford obtained his bachelor's in physics at Stanford University in 1994 and his doctorate in astronomy and instrumentation at Cornell in 2001. He held a Millikan postdoctoral fellowship at Caltech from 2001-2003 and has been on the science staff at JPL since that time. He has led the development and fielding of new submillimeter and millimeter-wave instrumentation on mountain-top site, using the datasets to study interstellar medium conditions in galaxies near and far. Current projects include a balloon-borne far-IR spectrometer, and on-chip mm-wave spectrometer, and a mm-wave line intensity mapping instrument. He is also invested in developing concepts and the necessary detector systems for cryogenic space far-IR astrophysics missions.

Damon Bradley is head of Instrument Electronics Development Branch and founder of the Digital Signal Processing Technology Group at NASA Goddard Space Flight Center, where he supervises the research and development work of more than 70 engineers and 30 projects. $\mathrm{He}$ is also co-investigator of three different spaceflight spectrometer systems and serves as an adjunct professor in the Electrical and Computer Engineering Department at UMBC.

Ruth Chiang Carter was a systems engineer and space flight mission development manager at the NASA Goddard Space Flight Center from 1986 to 2019 (retired, 2019). She managed and led a team to design and develop flight systems, including Hubble Space Telescope Servicing Missions, Tropical Rainfall Measuring Mission, and the Origins Space Telescope mission study. She received her BS and MS degrees in nuclear engineering from the University of Maryland at College Park.

Michael DiPirro-After receiving a $\mathrm{PhD}$ in low-temperature physics from the State University of NY at Buffalo, and a one-year NRC postdoctoral fellowship at NIST, he joined NASA Goddard in 1980. He has worked on a number of astrophysics missions over the last 40 years including COBE, ASTRO-E, -E2, and -H, XRISM, Spitzer, WIRE, WISE, and JWST. Between COBE and ASTRO-E, he was the PI on the Superfluid Helium On-Orbit Transfer Flight Demonstration, and Co-I on a Cross Enterprise Technology Development Program to develop a new type of adiabatic demagnetization refrigerator. He is currently the technical lead and chief technologist for the Origins Space Telescope study for the 2020 Astrophysics Decadal Survey. 
Matthew East innovates large optics as a lead opto-mechanical engineer at L3Harris Technologies in Rochester, New York. Since age one, he regularly attended telescope making conventions, learning about optical systems, fabrication, and metrology. He has patented inventions in additive manufactured optics and has published concepts for spaceborne astronomy missions. He holds a BSME from RPI and MSEM from Clarkson University. His goal is to drive the next generation of technology that enables breakthrough astronomical observations.

Kimberly Ennico has built infrared cameras, spectrometers, suborbital instruments, and lunar payloads. She has tested detectors at particle accelerators. She has served as SOFIA Project Scientist and New Horizons deputy project scientist. She has been a STDT member for Origins Space Telescope, authored 120+ peer-reviewed papers and delivered 50+ invited technical talks and 70+ public presentations. Asteroid 154587 Ennico is named for her. She is currently the VIPER lunar rover deputy project scientist.

Thomas P. Greene received his $\mathrm{PhD}$ in astronomy from the University of Arizona in 1991 and held positions at the University of Hawaii Institute for Astronomy and Lockheed Martin before joining NASA in 1998. He is an astrophysicist at NASA's Ames Research Center in Mountain View, California. He is a co-investigator on the JWST NIRCam instrument and a member of the JWST Mid-Infrared Science Team and conducts observational studies of young stars and extrasolar planets.

Joseph Howard received his BS degree in physics from the US Naval Academy in Annapolis, Maryland, and his PhD in optical design from The Institute of Optics, University of Rochester, in Rochester, New York. He now serves as an optical designer for NASA, working on projects including the James Webb Space Telescope, the Roman Space Telescope, Origins, and other future space missions.

John S. Knight is a senior staff consultant in Missions Systems Engineering at Ball Aerospace. His current focus is payload architecture for large space systems. He has been involved with segmented space telescopes for over $15+$ years concentrating on system engineering, modeling, test design for ground-based verification and flight commissioning.

Paul Lightsey has 50+ years' experience including work on HST instruments, Webb, and studies for future large space telescopes. He has co-authored a book on Systems Engineering of Astronomical Telescopes. He has a BS from Colorado State University, a PhD from Cornell University, and has received the Distinguished Public Service Medal from NASA. He is a SPIE fellow, and a senior member of OSA.

John C. Mather is senior project scientist for the James Webb Space Telescope. As a 28-yearold postdoc, he led the proposal for the Cosmic Background Explorer satellite (COBE) and served as its project scientist, leading the COBE team to success and to the Nobel Prize in physics (2006). He leads the science study team for the Orbiting Configurable Artificial Star, to enable high performance adaptive optics on ground-based telescopes.

Margaret Meixner is director of the SOFIA Science Mission Operations center. She holds bachelor's degrees in electrical engineering and math from the University of Maryland, College Park, and a PhD in astronomy from the University of California, Berkeley. Her research includes infrared instrumentation and dust evolution in galaxies. She is a member of the JWST/MIRI Science Team and community co-chair of NASA's STDT for the Origins Space Telescope mission concept. She is a former JWST Project Scientist and Distinguished Astronomer at STScI and a fellow of the AAAS.

Gary Melnick is a senior astronomer at the Harvard-Smithsonian Center for Astrophysics specializing in infrared/submillimeter spectroscopy and astrochemistry. For more than 45 years, he has conducted airborne, balloon-borne, and space-based observations focused on the main coolants of interstellar clouds and understanding the formation, abundance, and distribution of interstellar water. He was PI of the SWAS Explorer mission, Co-I on the Herschel mission, and science lead for the Ices Investigation on the upcoming SPHEREx mission. 
Stefanie Milam is an astrochemist with expertise in observations and spectroscopy at millimeter and sub-millimeter wavelengths and coordinating ground-based campaigns of cometary apparitions at multiple wavelengths. She also represents the planetary science community on a number of astrophysics missions and concepts including JWST, Roman Space Telescope, and the Origins Science and Technology Development Team.

Alexandra Pope holds a PhD from the University of British Columbia and is an associate professor of astronomy at the University of Massachusetts Amherst. She is an observational astronomer who specializes in infrared, (sub)millimeter and radio observations of dusty galaxies at high redshift.

Gerard Rafanelli received his $\mathrm{PhD}$ from Stevens Institute of Technology and is a Principal Engineering Fellow at Raytheon Technologies (RTX). As a result of his 36-year career in the aerospace and defense sector, he is a nationally recognized electro-optical systems expert for a broad range of platforms and missions. He has led multiple strategic initiatives for RTX space, air-, ground-, and sea-based sensors and weapon systems, including active and passive techniques.

Thomas Roellig has worked in the NASA civil service as an astrophysicist at the NASA Ames Research Center since 1980 and is currently the chief of the Astrophysics Branch at Ames. His scientific research interests have spanned a wide range of infrared astronomy and astronomical instrumentation development. He has conducted research and published papers in infrared instrument development, solar science, solar system science, star formation, interstellar medium, and brown dwarf astronomy.

Anita Sengupta is an aerospace engineer, entrepreneur, instrument-rated pilot, rocket scientist, and veteran of the space program. She has developed technologies that have enabled the exploration of deep space and novel transportation systems for over 20 years at NASA, Virgin, and Boeing. She is a research associate professor astronautics at the University of Southern California and a founding member of two electric aviation companies.

Kevin Stevenson is a staff astronomer at Johns Hopkins Applied Physics Laboratory and adjunct assistant professor in Johns Hopkins University's Department of Earth and Planetary Sciences. He is interested in characterizing the architectures and atmospheres of extrasolar planets to better understand their nature and origin; developing exoplanet mission concepts to measure the composition and chemical properties of nearby potentially habitable worlds; and building software packages and tools to enable exoplanet observations and atmospheric characterization.

Martina Wiedner is a CNRS research scientist at Paris Observatory. She received her undergraduate degree from Karlsruhe University and her PhD from Cambridge University. She held an SMA postdoc at the Harvard-Smithsonian Center for Astrophysics and a post at the University of Cologne. She built and designed several heterodyne receivers, including the 1.4-THz CONDOR receiver and $183-\mathrm{GHz}$ water vapor monitors. She is interested in star formation and starburst galaxies.

Chi Wu is a mission system engineer at NASA/GSFC with experiences ranging from communication satellites in private industry to NASA science missions, from preparing proposals in response to Announcements of Opportunity to conducting mission studies, mission planning, and mission development from pre-phase A to launch.

Biographies of the other authors are not available. 